\title{
É possível Recomendar Objetos de Aprendizagem Físico-Virtuais?
}

\author{
Gabriel Leitão $^{1}$ e Raimundo Barreto ${ }^{1}$ \\ ${ }^{1}$ Instituto de Computação - Universidade Federal do Amazonas (UFAM) \\ Av. General Rodrigo Otávio, 6200, 69077-000 - Manaus - AM - Brasil \\ \{gabriel.leitao, rbarreto\}@icomp.ufam.edu.br
}

\section{Introdução}

Garantir estudantes entusiasmados pela ciência e pela pesquisa científica requer iniciativas que os envolvam em experiências científicas interessantes e motivadoras. Hoje, os estudantes podem investigar fenômenos científicos, usando diversas ferramentas, técnicas, modelos e teorias da ciência, a partir de laboratórios físicos que suportam interações com o mundo material, ou em laboratórios virtuais que utilizam simulações. Mas, será que é possível unir as duas coisas? Isto é, unir o aspecto físico com o virtual de tal forma que haja comunicação do componente físico com o seu respectivo componente virtual e vice-versa? É possível que essa interação entre os componentes físicos e os componentes virtuais possa ser, de alguma forma, útil para o ensino-aprendizagem? Advogamos que é possível fazer ambos, ou seja, a investigação física, a investigação virtual e, além disso, oferecer recomendações para a combinação dos dois. Entretanto, há diversos desafios que ainda precisam ser superados. Alinhado com os sistemas físico-cibernéticos (cyber-physical systems), que integram sistemas computacionais com objetos do mundo físico, surgiu o conceito de objeto físico-virtual de aprendizagem, que é qualquer tipo de manipulativo que integre aspectos físicos e virtuais no mesmo recurso, que possua o intuito de auxiliar o processo de ensino-aprendizagem, e que possa ser reutilizado em diferentes contextos. Sistemas de recomendação são ferramentas automatizadas que usam algoritmos de filtragem para coletar dados e informações, a fim de realizar recomendações inteligentes, isto é, as mais relevantes, baseada no perfil, gostos e preferências de seus clientes. O foco desse artigo está em plataformas educacionais que usem tecnologia a partir de ambientes físico-cibernéticos, de tal forma que objetos físico-virtuais de aprendizagem possam ser integrados no ensino, que seja avaliada a sua eficácia e que possam ser geradas recomendações para os alunos.

\section{$\underline{\text { Desafios }}$}

Os grandes desafios, tanto para as atividades pedagógicas baseadas nos manipulativos físicos quanto nos virtuais, são: (i) como registrar as interações entre os estudantes e o ambiente físico-virtual, e como usar tais informações para avaliar a experiência de aprendizado e das condições do ambiente de ensino; (ii) como identificar as eventuais ações corretivas a serem tomadas; (iii) quais recomendações podem ser feitas para melhorar a qualidade da aprendizagem; (iv) como definir o progresso, ou falta dele; e, antes de tudo, (v) onde encontrar estes manipulativos e como utilizá-los de modo eficiente durante uma aula. Para tanto, dentro do contexto de ambientes físico/virtuais, ainda carecemos de métodos, técnicas e ferramentas que consigam medir o progresso de cada aluno individualmente e em grupo, bem como recomendar experimentos físico-virtuais para que um aluno venha a melhorar o seu desempenho acadêmico. Além disso, também há a ne- 
VIII Congresso Brasileiro de Informática na Educação (CBIE 2019)

Anais do VIII Workshop de Desafios da Computação aplicada à Educação (DesafIE 2019)

cessidade de ferramentas que tornem o uso e o reuso mais simples e de repositórios que facilitem o acesso aos objetos físico-virtuais já existentes.

\section{Importância e Benefícios}

Tanto os laboratórios físicos quanto os virtuais podem alcançar objetivos semelhantes, como explorar a natureza da ciência, desenvolver habilidades de trabalho em equipe, cultivar o interesse pela ciência, promover a compreensão conceitual e desenvolver habilidades de pesquisa. Usando equipamento físico, os alunos podem desenvolver habilidades práticas, incluindo a solução de problemas de configuração de equipamentos. Além disso, os laboratórios físicos podem tirar proveito de informações táteis que, de certa forma, ajudam a estimular o desenvolvimento do conhecimento conceitual. Por outro lado, os laboratórios virtuais podem simplificar os modelos de aprendizagem, removendo os detalhes menos importantes. Os alunos podem realizar experimentos sobre fenômenos não facilmente observáveis sem a necessidade de ter os respectivos objetos físicos, o que é mais fácil e mais barato a médio/longo prazo. Os laboratórios virtuais possibilitam que os alunos também possam vincular os processos físicos a equações matemáticas, o que possibilita fazer abstrações sobre diferentes representações físicas. Além disso, as experiências virtuais oferecem eficiência, pois normalmente exigem menos tempo de preparação e fornecem resultados praticamente instantâneos, enquanto que os experimentos físicos incluem atrasos reais e encorajam um planejamento cuidadoso.

É importante salientar que, nos últimos anos, o uso de sistemas ciberfísicos no contexto educacional tem sido objeto de diversas pesquisas fora do Brasil, onde alguns estudos tem implementado manipulativos físicos e virtuais em separado com a finalidade de realizar comparações [Zacharia and Olympiou 2011, Salehi et al. 2014], outros trabalhos mais recentes utilizam manipulativos híbridos (físicovirtuais) [Ha and Fang 2018, Azad and Hashemian 2016] ou utilizam físicos e virtuais concomitantemente [Blikstein et al. 2016]. De modo geral, o que se tem notado é que a associação entre o físico e o virtual tende a ajudar mais os processos de aprendizagem dos estudantes do que a utilização exclusiva de apenas um tipo de manipulativo.

\section{Formas de Suscitar Colaborações}

A principal forma de suscitar colaborações para trabalhar no desafio é através das discussões sobre este assunto. Acreditamos que o DesafIE é um veículo ideal para isso. Ademais, é preciso estabelecer diálogos e parcerias entre os grupos de pesquisa que tem atuado no desenvolvimento de estratégias educacionais que utilizem manipulativos tanto físicos quanto virtuais de modo a possibilitar tanto a criação de extensões para que estes manipulativos tenham suas partes físicas e virtuais integradas entre si, quanto o seu reuso.

\section{Dificuldades Atuais e Vindouras}

Algumas dificuldades potenciais inerentes ao desafio foram identificadas e estão descritas a seguir: (i) na base de dados do CBIE, de 2014 até o momento atual, há apenas dois artigos que tratam desse tema, onde o mais antigo propõe uma plataforma básica para implementação de sistemas físico-virtuais [Santos et al. 2014] e mais recente apresenta uma abordagem de leitura colaborativa em ambiente físico-virtual usando realidade aumentada [Imamura and Baranauskas 2018]; (ii) há um problema de se fazer recomendações e avaliações desses objetos físico-virtuais uma vez que não há um repositório dos mesmos; (iii) há um problema para a criação e descrição de objetos de 
VIII Congresso Brasileiro de Informática na Educação (CBIE 2019)

Anais do VIII Workshop de Desafios da Computação aplicada à Educação (DesafIE 2019)

aprendizagem físico-virtuais porque não há um padrão de metadados e/ou ontologias; e (iv) há uma problema de sistematização da execução desses objetos, visto que os objetos criados não estão integrados a nenhuma plataforma ou a quaisquer ambientes de aprendizagem.

\section{Ações para Enfrentar o Desafio}

Este é um tópico de pesquisa que precisa ser mais investigado, principalmente por causa dos benefícios propostos para o ensino-aprendizagem. Além disso, para se enfrentar o desafio, precisamos discutir como gerar repositórios de objetos de aprendizagem físico-virtuais para servir como base para a recomendação desses objetos diante de uma situação de desempenho fraco em um assunto. Adicionalmente, é preciso estender os padrões de objetos de aprendizagem (provavelmente o OBAA) no sentido da geração de novos metadados e/ou ontologias específicas para tais objetos. E, finalmente, precisamos definir como deve ser a execução os objetos físico-virtuais em sala de aula/laboratório, ressaltando o aspecto de execução simultânea (físico e virtual) e da comunicação.

\section{Agradecimentos}

Esta pesquisa, conforme previsto no Art. 48 do decreto $\mathrm{n}^{\circ}$ 6.008/2006, foi financiada pela Samsung Eletrônica da Amazônia Ltda, nos termos da Lei Federal nº 8.387/1991, através de convênio $\mathrm{n}^{\circ}$ 003, firmado com o ICOMP/UFAM, e pela Fundação de Amparo à Pesquisa do Estado do Amazonas (FAPEAM) através do projeto 22/2018 (Edital UNIVERSAL).

\section{Referências}

Azad, A. and Hashemian, R. (2016). Cyber-physical systems in stem disciplines. In Proceedings of 2016 SAI Computing Conference, SAI 2016, pages 868-874. Institute of Electrical and Electronics Engineers Inc. cited By 1.

Blikstein, P., Fuhrmann, T., and Salehi, S. (2016). Using the bifocal modeling framework to resolve "discrepant events" between physical experiments and virtual models in biology. Journal of Science Education and Technology, 25(4):513-526.

Ha, O. and Fang, N. (2018). Interactive virtual and physical manipulatives for improving students' spatial skills. Journal of Educational Computing Research, 55(8):10881110 .

Imamura, R. E. and Baranauskas, M. C. (2018). Criando uma experiência de leitura colaborativa de histórias fictícias físico-virtuais com realidade aumentada. In Brazilian Symposium on Computers in Education (Simpósio Brasileiro de Informática na Educação-SBIE), volume 29, page 31.

Salehi, S., Schneider, B., and Blikstein, P. (2014). The effects of physical and virtual manipulatives on learning basic concepts in electronics. In CHI' 14 Extended Abstracts on Human Factors in Computing Systems, CHI EA '14, pages 2263-2268, New York, NY, USA. ACM.

Santos, R., Botelho, S., and Bichet, M. (2014). Ambientes físico-virtuais de aprendizagem. Simpósio Brasileiro de Informática na Educação, 25(1):70.

Zacharia, Z. C. and Olympiou, G. (2011). Physical versus virtual manipulative experimentation in physics learning. Learning and Instruction, 21(3):317 - 331. 